\section{SOBRE A ORIGEM INCOMUM DA ARTERIA TESTICULAR EM FETOS DE BOVINOS AZEBUADOS*}

\section{FREDERICO DZANAM CARNEIRO E SILUA} Professor Adjunto Universidade Federal de Uberlandia - MG

PEDRO PRIMO BOMBONATO Professor Assistente Doutor Faculdade de Medicina Veterinaria e Zootecnia da USP

\section{RENATO SOUTO SEVERINO} Professor Adjunto

Universidade Federal de Uberlandia - MG

JOSE PEDUTI NETO Professor Adjunto Faculdade de Medicina Veterinária e Zootecnia da USP

SILUA, F.D.C.; BOMBONATO, P.P.: SEVERINO, R.S.; PEDUTI NETO, J. Sobre origem incomum da apteria testicular em fetos de bovinos azebuados. Rev.Fac.Med.Vet.Zootec. Univ.5.Paulo, 24 (1):13-15, 1987.

RESUMO: Estudando o comportamento dos ramos viscerais do tracto abdaminal da arteria aorta roi encantrado um caso de origem incomum da arteria testicular.

UNITERMOS: Anatomia, bovinos; Arterias; Testiculos; Bovinos, $r$ aca Zebu

\section{INTRODUCAO E LITERATURA}

0 conhecimento mais apurado da morfologia dos bovinos de racas indianas vem se constituindo em uma das metas prioritbrias dentro das linhas de pesauisa, desenvolvidas na arrea ae Anatomia Descritiva e Topografica desta Faculdade. Assim, em recente estudo, SILUA et al $i j, 11$ (1985) propuseram-se a estudar os colaterais viscerais da arteria aorta pars abdominalis, particularmente do comportamento das Aa. mesentericas cranjais e caudais em fetos de animais azebuados. En tal oportunidade, conforme exposto em outra publicacă ( 5 ILUA et alii, 12. 1985), encontramos nesse material, a emergencia das arterias ovaricas direita e esquerda, a partir da $A$. mesenterica cranial, antes da emissao da $A$. colica esquerda pela face cranioventral. Continuando as preparacơes, com vistas ao objetivo primeiro e, inclusive, com a coleço de novas racas, tivemos o ensejo de surpreender para a $A$. testicular, variacáes de natureza semelhante que julgamos interessante relatar, face a escassez de informacoles quanto ao assunto, na literatura consultada.

De fato, os autores classicos limitamse a descrever, de modo geral, a A. testicular como ramo da A. aorta abdominal (f ace ventral) e, ainda mais, para o eqüino, năo apontado diferencas notaveis para os bovinos, a maneira de BOSSI, 1 (s.d.), BRUNI \& ZIMMERL, 2 (1947), ELLENBERGER \&'BAUM, 3 (1332), LESBRE, 7 (1923) e tambem por MONTANE \& BOURDELLE, 8 (1917), embora dediquem um dos tomos de sua obra, especialemente aos ruminantes; as consideracöes sao identicas para os dados fornecidos por GETTY, 4 (1981) e KOCH, 6 (1965)., em cap tulo proprio aos bovinos e dor GODINHD et alij, 5 (1981) em compendio de anatomia dos ruminantes.

Por outro lado, 5CHWARZE \& SCHRÖDER, 10 (1972) aludem a posslveis alteracós quanto a origem da A. testicular, isto e, da oropria aorta, a diferentes distancias daquele da $A$. mesenterica caudal, desta mesma ou da A. renal; entretanto, fazem-no de forma poued clara, a deixar duvidas se tais disposicäes podem ser generalizadas, ou se sao proprias dos equinos ou dos carnlvoros, pois nada particularizam ao cuidar dos bovinos.

Finalmente, cabe lembrar a divergencia de nomenclatura, encontrada entre os AR., ao referirem-se a arteria em estudo, vale dizer, arteria espermatica interna (ELLENBERGER \& BAUM, 3, 1932, KOCH, 6, 1965, SCHWARZE \& SCHRODER, 10, 1972) arteria espermatica interna ou arteria grande testicular (BOS5I, 1, 5.D., BRUNI \& ZIMMERL, 2 , 1951, LESBRE, 7, 1923, MONTANE \& BOURDELLE, 8,1917 ), ou arteria testicular (GETTY, 4, 1981 e GODINHO et alii. 5, 1981), designaço tambem por nos ja adotada ( ILUA et alii, 11, 12, 1985) e agora mantida, inclusive por ser a recomenoada dela

Trabalho apresentado ao 20. Congresso Brasileiro de Medicina Veterinária. Cuiabá, 1986 
14 SILVA, F.O.C.; BOMBONATO, P.P.; SEVERINO, R.S.; PEDUTI NETO, J.

NOMINA ANATOMICA VETERINARIA, 9, 1983. MATERIAL E METODO

Baseia-se, este relato no exame de 16 fetos de bovinos azebuados, englobando os 15 ja utilizados na referida pesquisa sobre - comportamento das arterias mesentericas e sempre procedentes do municlpio de Uberaba, Estado de Minas Gerais (Frigorlfico Triangulo). O material recolhido era congelado $e$ remetido ao Laboratorio de Anatomia Descritiva e Topográfica da Faculdade de Medicina Veterinaria e Zootecnia da Universidade de 5ro Paulo. Aqui em concordancia as tecnicas utilizadas para o primeiro bloco, procedlamos, de inicio, ao descongelamento em agua corrente e mediamos os animais, tomando igualmente como pontos de reparo, eranial e caudal, a crista nucal e a articulacรo entre a primeira e a segunda vertebra cocclgea, obtendo para o expecime ora estudado, o valor de 45,5 cm compreendido, portanto, entre os limites máximo e mlnimo anotados para aqueles, ou seja, $31,5 \mathrm{~cm}$ e $67,5 \mathrm{~cm}$, permitindo-nos, assim, este agrupamento de tal sorte a contarmos, agora, com $16^{\circ}$ fetos machos. Na mesma sequencia da pesquisa anterior, obtinhamos as preparaces abrindo a cavidade toracica, ao nlvel do nono espaco intercostal do lado esquerdo para, com solucăo de Neoprene latex "450", acrescida de corante especificico verde*, injetarmos, em sentido caudal, a R. aorta, a partir de sua pars thoracica, seguindo-se fixacro em solucro aquosa de formol a $10 \%$ e disseccro dos vasos de nosso interesse, depois de aberta a cavidade abdominal por duas inciszos, uma de sentido dorsoventral e tangente ao bordo caudal do altimo arco costal outra, sobre a linha alba, em toda sua extensgo. Finalmente, com vistas à documentacgo, fotografamos a peca.

\section{RESULTADOS}

Dos 16 fetos estudados, surpreendeunos um no qual a $A$. tejticular esquerda destacava-se pelo inusitado de sua origem. no foto, tal vaso nascia da $A$. mesenterica caudal, mais exatamente, na face cauds!ateral de seu terco oroximal. A A. tesicicular direita mostrava a derivacgo usualmente conhecida, isto e, Drocedia diretamente da pars abdominalis da $A$. aorta, em sua face ventral. Afora tal caráter de excecao, apresentado pela h. testicular esquerda, exibiam ambos o comportamento classicamente descrito.

\section{COMENTARIOS E CONCLUSOES}

De infcio, Darece-nos interessante lembrar as diferencas apresentadas pelos autores, quanto à designaça da arteria estudada, vale dizer. A. espermatica inter- na, Dara alguns (ELLENBERGER \& BAUM, 3 , KDCH, 6. S[HWARZE \& SCHRÓDER, 10), A: espermatica interna ou grande testiculap, para outros (BDSSI, 1, BRUNI \& ZIMMERL, 2 , LESBRE, 7, e MONTENE \& BOURDELLE, 8) e, por fim, artéria testicular (GETTY, 4, GODINHO et alii, 5); esta ultima denominaça tambem foi por nds perfilhada, em especial dor ser a indicada, atualmente, pela NOMINA ANATDMICA VETERINARIA, 3. Diga-se, de Dassagem, ngo ter a consideraço feita, carater critico ou de confronto, particularmente pelas seguidas modificacales introduzidas na nomenclatura anatomica, mas ao contrário e por estefato, advertir o eventual leitor sobre posslveis divergencias neste campo. Mais que isso, cremos relevante lembrar a norma usualmente seguida pelos autores classicos de oferecerem, em anatomia veterinaria, descriçes padrgo para o eqoino, generalizando-as indiscriminadamente, para as outras especies. inclusive os bovinos, como, no caso, fazem BOSSI, 1, BRUNI \& ZIMMERL，2, ELLENBERGER \& BAUM, 3, LESBRE, 7, ou mesmo quando cuidam desses animais em capitulo ou termo especifico, haja visto. respectivamente, GETTY, 4 e KOCH, 6, entre os primeiros, e MONTANE \& BOURDELLE, 6. Por outro lado, nao cabe, de modo geral, cotejo de nosso achado com as informaçres obtidas, pois alem dos já citados fatores, eximem-se os tratadistas de esclarecimentos quanto a idade e raca dos animais observados. liaitando-se como jà expusemos, a indicar a emergencia da artéria testicular a a partir da arteria aorta - pars abdominalis.; - as ultimas seadencias repetem-se ao considerarmos GDDINHO et alii, 5, embora seu compendio refira-se aos ruminantes domesticos. Ressalve-se, entretanto, ser valido, em publicacies dessa ordem, o criterio de apresentar o usual, o mais comum. Quanto a SCHWAR?F, \& SCHRDDER, 19: resta-nos relembrar que, embora indicando possiveis alteracales quanto ao ramo de origem da arteria testicular, o fazem de maneira pouco explicita e dirlamos mesmo, a permitir interpretacós várias relativamente à especie considerada, nada registrando ao levar em conta os bovinos.

Particularizando, agora, os resultados otijus no material recoinido e, eni parte utilizados por SILUA et alii, 11, ao analisar a origem e comootamento de outros colaterais aóticos, tais sejam as arterias mesentéricas cranial e caudal, Darece-nos interessante destacar a ocorpencia, ainda que excepcional, mas semelhante, de variacores na emergencia das arterias destinadas as gonadas, seja, os ovarios, conforme descrica anterior de SILUA et alii, 12 , sejam os testiculos, mais exatamente esquerdo, como surpreendemos desta feita. Acentuamos, aqui, tal relacionamento, por se tratar de material homogeneo, ou seja, fetos de bovinos azebuados procedentes de una mesma regigo de pecuária. 
Finalmente, cumpre-se lembrar que achados como estes, embora ocasionais, vem reforcar a proposica feita, neste Departemento de Anatomia, da necessidade premente de melhor conhecer a morfologia dos bovinos de racas indianas, especialmente pelo papel por elas desempentiado na criacra nacional.
SILVA, F.O.C.; BOMBONATO, P.P.: SEVERINO, R.S.; PEDUTI NETO, J. A case of uncommon origin of the arteria testicularis in zebu eattle. Rev.fac.Med.Vet.Zootec. Univ.S.Paulo, 24(1): 13-15, 1987.

SUMMARY: There was deatched a case of uncommon origin of the arteria testicularis, from a study related to the origin ond pattern of the visceral branches of the arteria aorta - pars testicularls in lebu foetuses.

UNITERMS: Anatomy of cattle; Arteries; Testicles; Zebu catile

\section{REFERENCIAS BIBLIDGRAFIAS}

1 - B055I, $U$. Angiologia. In: B0S5I, U.; CARADONNA, G.F.; SPAMPANI, G.; VARALDI, L.; ZIMMERL, U. Trattato di anatomia veterinaria. Milano, Francesco Vallardi, s.d. v.2, p.210213 .

2 - BRUNI, A.C. \& ZIMMERL, U. Anatomia degli animali domestici. 2.ed. Milano, Francesco Vallardi, 1951. v. 2, p. 352 .

3 - ElLENBERgER, w \& BRUM, H. Handbuch der vergleicenden Anatomie der Haustiere. 18. auf. Berlin, Julius Springer, 1932. v.8, p.707.

4 - GETTY, R. Anatomia dos animais domésticos. Trad. de Sisson and Grossman's The anatomy of domestic animals. 5.ed. Rio de Janeiro, Interamericana, 1981.v.1, p. 954.

5 - GODINHO, H.P.; CARDOSO, F.M.; NASCIMENTO, J.F. Anatomia dos ruminantes domesticos. Belo Horizonte, Instituto de Ciencias Biologicas da U.F.M.G. - Departamento de Morfologia, 1981. p. 173.

6 - KOCH, T. Lehrbuch der Veterinsr Anatomie. Jena, Gustav Fischer, 1965. v. 3, p.120-124.

7 - LESBRE, F.X. Précis d'anatomie comparée des animaux domestiques. Paris, J.B. Baillière, 1923. v.2. p. 367 .
8 - montane, L. \& BOURDelle, e. Anatomie regionale des animaux domestiques. Paris, J.B. Bailliere, 1917.v.2, p. 285 .

9 - NOMINA ANATOMICA VETERINARIA. 3.ed. Ithaca, International Committe on VeterinaryGross Anatomical Nomenclature, 1983.

10 - 5CHWARZE, E \& SCHRÖDER, L. Compendio de anatomia veterinaria. Taragoza, Aeribia, $19 \%<, v .3, p .7 \ddot{z}-73$.

11 - SILUA, F.O.C.; PEDUTI NETO, J.; BOMBONATO, P.P. Contribuica ao estudo da origem e ramificaca das arterias mesentericas cranial e caudal. em fetos de bovincs azebuados. Rev.Fac. Med.Vet. Znoiec. Uriv S.Pau$10,22: 31-36$, is65.

12 - SILUA, F.O.C.; BOMBONRTO, P.P.; RODRI GUES, C.A.; PEDUTI NETO, J. Sobre a origem incomum das arterias ovaricas em feios de bovinos azebuados. Rew.Fac.Med.Vet.Zootec. Univ. 5.Paulo, 22:65-66, 1985 .

Recebido para publicaczo em $27 / 08 / 85$ Aprovado para publicaço em $24 / 09 / 86$ 